Pacific Journal of Mathematic 


\section{STRONG COMPLETENESS IN PROFINITE GROUPS}

\section{ANDREW Pletch}

A profinite group is strongly complete if every subgroup of finite index is open. In this paper it is shown that a profinite group with finitely generated $p$-Sylow subgroups is strongly complete and that if $G$ is a finitely generated strongly complete profinite group and $A$ is a finitely generated pseudocompact $G$-modulo then any extension of $A$ by $G$ is strongly complete.

The purpose of this paper is to extend some results of Anderson [1] in the theory of strong completeness of profinite groups. A profinite group is a topological group whose topology is Hausdorff, compact and has neighborhood base of the identity consisting of certain subgroups of finite index. A profinite group is strongly complete if every subgroups of finite index is open. Since all open subgroups are also closed, a strongly complete profinite group has no dense subgroups of finite index except itself.

Our first result is:

THEOREM 1. Let $G$ be a profinite group, $G_{p}$ a p-Sylow subgroup, $U \unlhd G$ with $(G: U)=n<\infty$. $U$ is open in $G$ if and only if $U \cap G_{p}$ is open in $G_{p}$ for every prime $p$ which divides $n$.

CoROLlaRy 1. Let $G$ be a profinite group all of whose p-Sylow subgroups are finitely generated. Then $G$ is strongly complete.

Our second result is:

TheOREM 2. Let $A \nrightarrow E \rightarrow G$ be a short exact sequence of profinite groups. If $G$ is a finitely generated strongly complete profinite group and $A$ is a finitely generated pseudocompact $\hat{Z}[[G]]-$ module then $E$ is strongly complete.

CoRollary 1. Let $A \nrightarrow E \rightarrow G$ be a short exact sequence of profinite groups where $G$ is as in the theorem and $A$ contains a finite sequence of subgroups which are normal in $E$ : $A=A_{0} \geqq$ $A_{1} \geqq \cdots \geqq A_{n}=(e)$ such that $A_{i} / A_{i+1}$ is a finitely generated pseudocompact $\hat{Z}[[G]]-$ module for $i=0, \cdots, n-1$. Then $E$ is strongly complete.

In this paper all groups are profinite, all subgroups are closed, and all homomorphisms are continuous unless otherwise stated. We 
will call a proper subgroup of finite index large.

1. For any group, $G, x \in G$, the closed subgroup generated by $x, \overline{\langle x\rangle}$, is cyclic and so there is a continuous homomorphism $\rho$ : $\hat{\boldsymbol{Z}} \rightarrow \overline{\langle x\rangle}$ defined by $\rho(\lambda)=x^{\lambda}$. Writing $\hat{\boldsymbol{Z}}$ as $\Pi_{p} \hat{\boldsymbol{Z}}_{p}$, the product over all primes $p$ of $p$-adic integers, and then as $\hat{Z}_{p} \times \prod_{q \neq p} \hat{Z}_{q}$ and allowing the generator of $\hat{\boldsymbol{Z}}_{p} \times(0)$ to be $(1,0)$ and the generator of $(0) \times$ $\prod_{q \neq p} \hat{Z}_{q}$ to be $(0,1)$ one sees that $\overline{\left\langle x^{(1,0)}\right\rangle}$ is the $p$-Sylow subgroup of $\overline{\langle x\rangle}$ and $\overline{\left\langle x^{(0,1)}\right\rangle}$ its $p$-complement. Finitely generated pro-abelian groups are known to be strongly complete. Hence any homomorphism from $\overline{\langle x\rangle}$ to a finite group is continuous. With this we prove:

Proposition 1. Let $U$ be a large normal subgroup of $G, U$ not necessarily open, $x \in G$ such that $\bar{x} \in(G / U)_{p}, \quad p$-Sylow subgroup of $G / U$. Then $\overline{x^{(1,0)}}=\bar{x}$ in $G / U$.

Proof. The morphism $\overline{\langle x\rangle}$ to $\langle\bar{x}\rangle \leqq G / U$ is continuous as we have noted. $\langle\bar{x}\rangle$ is a finite cyclic $p$-group. Since $x=x^{(1,0)} \cdot x^{(0,1)}$ and $x^{(0,1)}$ is an element of $G$ whose order is prime to $p$, its image $\langle\bar{x}\rangle$ is the identity. Hence

$$
\bar{x}=\overline{x^{(1,0)} \cdot x^{(0,1)}}=\overline{x^{(1,0)} \cdot x^{(0,1)}}=\overline{x^{(1,0)}} .
$$

We call an element of $G$ a $p$-element if it belongs to some $p$ Sylow subgroup of $G$. For all $x$ in $G, x^{(1,0)}$ is a $p$-element and $x$ is a $p$-element if and only if $x=x^{(1,0)}$ (see [4]).

A net of elements $\left\{x_{\alpha}\right\}$ of a profinite group $G$ converges to an element $x$ if for all open normal subgroups $V$ of $G, x_{\alpha} V=x V$ for almost all $\alpha$.

Proposition 2. Let $\left\{x_{\alpha}\right\}$ be a net in $G$ converging to a p-element $x$. Then $\left\{x_{\alpha}^{(1,0)}\right\}$ is a net of p-elements which also converges to $x$.

Proof. If $x$ is a $p$-element then for any open normal subgroup $V$ of $G, x V$ is a $p$-element in $G / V$. By Proposition $1, x_{\alpha} V=$ $x_{\alpha}^{(1,0)} V$ if $x_{\alpha} V=x V$. The set $\left\{x_{\alpha}^{(1,0)}\right\}$ is clearly a net and hence the result.

Before proving Theorem 1 we need the following lemma.

LEMMA 1. Let $U \unlhd G, U$ not necessarily closed, such that for some p-Sylow subgroup $G_{p}$ of $G, U \cap G_{p}$ is closed in $G_{p}$. The set of all p-elements in $U$ is closed in $G$. 
Proof. Let $U_{p}=U \cap G_{p}$. The set of all $p$-elements in $U$ is

$$
\bigcup_{x \in G} U \cap G_{p}^{x}=\bigcup_{x \in G_{p}} U_{p}^{x}
$$

since $U$ is normal. Consider the function $U_{p} \times G \rightarrow G$ defined by $(u, g) \rightarrow g^{-1} u g$. Since $U_{p}$ is closed in $G_{p}$ it is compact and hence the function, which is easily continuous, is a closed function. Its image, which is precisely the set of $p$-elements of $U$, is therefore closed in $G$.

Proof of Theorem 1. Let $U \unlhd G$ of finite index. If $U$ is open then $U \cap G_{p}$ is open in $G_{p}$ for all $G_{p}$. Conversely suppose there exists large $U$ not open, the quotient group $\bar{U} / U$ has a nontrivial $p$-Sylow subgroup for some prime $p$. Hence there exists $x \notin U$ such that $\bar{e} \neq \bar{x} \in \bar{U} / U$ is a nontrivial $p$-element. By Proposition 1 we may assume $x$ is a $p$-element of $G$. Since $x \in \bar{U}$ there is a net $\left\{x_{\alpha}\right\}$ of elements of $U$ which converges to $x$. By Proposition 2, the net $\left\{x_{\alpha}^{(1,0)}\right\}$ also converges to $x$. Clearly, $x_{\alpha} \in U$ then $x_{\alpha}^{(1,0)} \in U$ by the strong completeness of $\overline{\left\langle x_{\alpha}\right\rangle}$. Hence the net $\left\{x_{\alpha}^{(1,0)}\right\}$ is a net of $p$ elements in $U$ which converge to a $p$-element $x$ not in $U$. By hypothesis and Lemma 1 , the set of $p$-element of $U$ is closed in $G$. Hence $x$ must be a $p$-element of $U$, contradiction.

Proof of Corollary 1 to Theorem 1. Finitely generated pro-pgroups are strongly complete, [1], [6]. Hence if $U \unlhd G, U$ large then $U \cap G_{p}$ is large in $G_{p}$ and so open. Therefore the theorem applies.

The above corollary is another proof of the result due to Oltikar and Ribes, [5], that finitely generated prosupersolvable groups are strongly complete since in the same paper they prove that such groups have finitely generated $p$-Sylow subgroups.

2. In this section we first show that the completed group algebra $\hat{Z}[[G]]$ (which we denote by $\Delta$ ) for a finitely generated profinite group, $G$, is in some sense strongly complete. Let $\operatorname{Mod}(G)$ be the category of $G$-modules, $G$ considered as an abstract group.

Proposition 3. Let $G$ be a finitely generated profinite group, $A \leqq \Delta$ such that $\Delta / A$ is finite and $A \in \operatorname{Mod}(G)$. Then $A$ is open in the topology of $\triangle$.

Before proving Proposition 3 we first review the topological structure of $\Delta$. 


$$
\Delta \simeq \lim _{n, \bar{U} \text { open }} Z / n \boldsymbol{Z}(G / U)
$$

A neighborhood base of (0) consists of the kernals, $\pi_{n, U}$ of the continuous morphisms $\Delta \rightarrow \boldsymbol{Z} / n \boldsymbol{Z}(G / U)$. In [2], Brummer notes that $\pi_{n, U}$ is the closed ideal generated by $\{(u-1) \mid u \in U\}$. In fact, as a pseudocompact $\Delta$-module, $\pi_{n, U}$ is precisely $\overline{n \Delta+\sum \Delta\left(u_{i}-1\right)}$ where $\left\{u_{i}\right\}$ is a set of topological generators of $U$. Therefore if $G$ and hence $U$ is finitely generated $\boldsymbol{I}_{n, U}$ is a finitely generated pseudocompact $\Delta$-module.

Proof of Proposition 3. Since $\Delta / A$ is finite, there exists $n$ such that $n \Delta \leqq A$. As well, $\Delta / A$ is trivial $U$-action for some large but not necessarily open subgroup $U$ of $G$. However $U$ contains the topological generators $\left\{u_{1}, \cdots, u_{s}\right\}$ of $\bar{U}$, its closure in $G$. In this case $\boldsymbol{I}_{n, \bar{U}}=\overline{n \Delta+\sum_{i=1}^{s} \Delta\left(u_{i}-1\right)}=n \Delta+\sum_{i=1}^{s} \Delta\left(u_{i}-1\right)$ and since clearly $B=\sum_{i=1}^{s} \Delta\left(u_{i}-1\right) \leqq A$ one has $I_{n, \bar{U}} \leqq A$ which implies $A$ is open as well.

The category of pseudocompact $\Delta$-modules, $P C_{G}^{p}$, is studied by Brummer, [2], and in the thesis of Gabriel. These modules are inverse limits of finite discrete $G$-modules with the corresponding profinite topology. If $M \in P C_{G}^{p}$ and $M$ is (topologically) finitely generated then $M$ is the continuous homomorphic image of $\bigoplus^{m} \Delta$, for some finite $m$.

CoRollary 1. Let $G$ be a finitely generated profinite group, $M \in P C_{G}^{p}, M$ finitely generated. If $A \leqq M$ such that $M / A$ is finite and $A \in \operatorname{Mod}(G)$, then $A$ is open in $M$.

Proof. If $\pi: \bigoplus^{m} \Delta \rightarrow M$ is defined, which is the case for $M$ finitely generated by at most $m$ elements, then one easily shows $\pi^{-1}(A)$ open in $\bigoplus^{m} \Delta$ and hence $A$ is open in $M$.

We now prove Theorem 2 .

Proof of Theorem 2. If $U$ is a large normal subgroup of $E$ but not necessarily open, its image in $G$ is open since $G$ is strongly complete and $U \cap A$ is open in $A$ by Corollary 1 to Proposition 3 since $U \cap A$ is preserved under the action of $G$ and hence belongs to $\operatorname{Mod}(G)$.

Consider the following commutative diagram of profinite groups

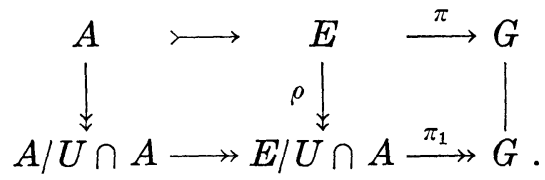


Clearly $\rho^{-1}(\rho(U))=U$ and $\rho$ is continuous so it suffices to show $\rho(U)$ is open or closed in $E / U \cap A$.

However, $\pi_{1}$ is a monomorphism when restricted to $\rho(U)$ and $\pi_{1} \circ \rho(U)$ is open in $G$. Therefore, restricted to $\pi_{1} \circ \rho(U), \pi_{1}$ has an inverse $\pi_{1}^{-1}$, such that $\pi_{1} \circ \pi_{1}^{-1}=1_{\pi_{1} \circ \rho(U)}$ and $\pi_{1}^{-1} \circ \pi_{1}=1_{\rho(U)}$. Hence there is a topology which we can place on $\rho(U)$ to make it a profinite group. Namely, $V \leqq \rho(U)$ is open iff $\pi_{1}(V)$ is open in $G$. But this is clearly the original relative topology on $\rho(U)$. We argue as follows: Let $V \leqq E / A \cap U$ be open in $E / A \cap U$. Then $\pi_{1}(V \cap \rho(U))$ is open in $G$. Hence $V \cap \rho(U)$ is open in $\rho(U)$ equipped with its profinite topology.

Hence the profinite topology of $\rho(U)$ is finer than its relative topology. Conversely, if the profinite topology is properly finer then we extend this topology to a profinite topology on $E / A \cap U$. Hence $E / A \cap U$ can be equipped with two profinite topologies, one coarser than the other and this is impossible. Hence the two topologies on $\rho(U)$ are identical so that $\rho(U)$ is closed in $E / A \cap U$ since it is compact. Hence the result.

Proof of Corollary 1 to Theorem 2. The profinite group, $E$, of Theorem 2 is finitely generated. By the Theorem, $E / A_{1}$ is strongly complete. By induction, if $E / A_{i}$ is strongly complete then the short exact sequence $A_{i} / A_{i+1} \nrightarrow E / A_{i+1} \rightarrow E / A_{i}$ shows $E / A_{i+1}$ to be strongly complete. Hence, by induction, the corollary holds.

Finally we notice that in the case $A \nrightarrow E \rightarrow G$ verifies the hypothesis of Corollary 1 to Theorem 2 then $E$ is finitely generated.

Proposition 4. Let $A \nrightarrow E \rightarrow G$ be a split short exact sequence of profinite groups where $E$ is generated by $n$ elements and $A$ is abelian. Then $A$ is a pseudocompact $\Delta$-module generated by $n$ elements.

Proof. A similar results is proved by Hartley, [3, Lemma 5] for finite groups and easily carries over to profinite groups.

Corollary 2 to TheOREM 2. If $A \nrightarrow E \rightarrow G$ is a split short exact sequence of profinite groups such that $E$ is finitely generated, $A$ is abelian and $G$ is strongly complete, then $E$ is strongly complete.

Proof. Proposition 4 allows us to say $A$ is a finitely generated pseudocompact $G$-module and so we may apply the theorem. 


\section{REFERENCES}

1. M. P. Anderson, Subgroups of finite index in profinite groups, Pacific J. Math., 62 (1976), 19-28.

2. A. Brummer, Pseudocompact algebras, profinite groups and class formations, J. Algebra, 4 (1968), 442-470.

3. B. Hartley, Subgroups of finite index in profinite groups, Math. Zeit., 168 (1979), 71-76.

4. W. N. Herfort, Compact torsion groups and finite exponent, Achiv. der Mathematik, 33 (1979), 404-410.

5. B. C. Oltikar and L. Ribes, On prosupersolvable groups, Pacific J. Math., 77 (1978), 183-188.

6. J. P. Serre, Letter to the author dated March 26, 1975.

Received November 11, 1980 and in revised form March 9, 1981. The work on this paper was partially supported by FAPESP Grant no. 78/0952.

INSTITUTO DE MATHEMATICA E ESTATISTICA

UNIVERSIDADE DE SÃO PAULO

São PaUlo, Brasil

Current address: Depto Mathematicas

Universidad de Puerto Rico

Mayaguez, PR 00708 


\section{PACIFIC JOURNAL OF MATHEMATICS}

\section{EDITORS}

DONALD BABBITT (Managing Editor)

University of California

Los Angeles, California 90024

Hugo RossI

University of Utah

Salt Lake City, UT 84112

C. C. MOORE and ANDREW OGG

University of California

Berkeley, CA 94720

\section{J. DugundJI}

Department of Mathematics University of Southern California Los Angeles, California 90007

R. Finn and J. Milgram Stanford University

Stanford, California 94305

\section{ASSOCIATE EDITORS}
R. ARENS
E. F. BeCKENBACH
B. H. Neumann
F. WoLF
K. YoSHIDA

\section{SUPPORTING INSTITUTIONS}

UNIVERSITY OF ARIZONA

UNIVERSITY OF BRITISH COLUMBIA

CALIFORNIA INSTITUTE OF TECHNOLOGY

UNIVERSITY OF CALIFORNIA

MONTANA STATE UNIVERSITY

UNIVERSITY OF NEVADA, RENO

NEW MEXICO STATE UNIVERSITY

OREGON STATE UNIVERSITY
UNIVERSITY OF OREGON

UNIVERSITY OF SOUTHERN CALIFORNIA

STANFORD UNIVERSITY

UNIVERSITY OF HAWAII

UNIVERSITY OF TOKYO

UNIVERSITY OF UTAH

WASHINGTON STATE UNIVERSITY

UNIVERSITY OF WASHINGTON 


\section{Pacific Journal of Mathematics}

\section{Vol. 97, No. $1 \quad$ January, 1981}

Charles A. Asmuth and Joe Repka, Tensor products for $S L_{2}(\mathfrak{k})$. II.

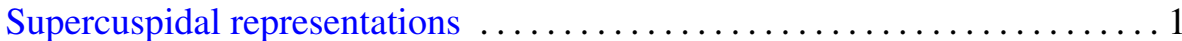

Joseph Barback, On finite sums of regressive isols . ................. 19

Matthew G. Brin and Daniel Russell McMillan, Jr., Generalized

three-manifolds with zero-dimensional nonmanifold set ............29

Kun Soo Chang, Converse measurability theorems for Yeh-Wiener space . . . 59

Christopher Brian Croke, A "maximal torus" type theorem for complete

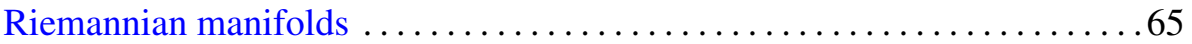

Gustave Adam Efroymson, Sums of squares in planar Nash rings . . . . . . 75

John Robert Fisher, Axiomatic radical and semisimple classes of rings . . . .81

Betty Kvarda, Consecutive integers for which $n^{2}+1$ is composite .......93

Roosevelt Gentry, New diagram proofs of the Hausdorff-Young theorem

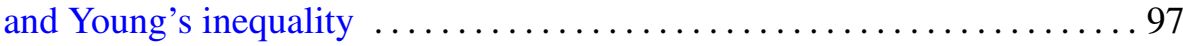

Patrick M. Gilmer, Topological proof of the $G$-signature theorem for $G$

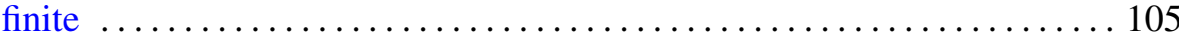

Chung Wei Ha, A noncompact minimax theorem .................. 115

James J. Hebda, Manifolds admitting taut hyperspheres ................ 119

Takayuki Kawada, Sample functions of Pólya processes ............. 125

Peter K. F. Kuhfittig, Common fixed points of nonexpansive mappings by

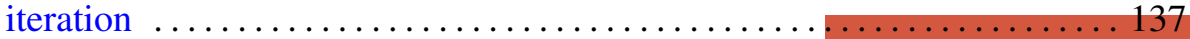

James Thomas Loats and Judith Roitman, Almost rigid Hopfian and dual

Hopfian atomic Boolean algebras .......................... 141

Roger McCann, On embedding semiflows into a radial flow on $l_{2} \ldots \ldots \ldots 151$

John McDonald, Closed orbits of convex sets of operators on the disk

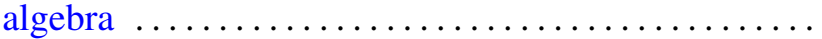

Mark D. Meyerson, Convexity and the table theorem .............. 167

Arnold William Miller, Generic Souslin sets . . . . . . . . . . . . . . . . 171

Takemi Mizokami, On the closed images of paracomplexes $\ldots \ldots \ldots \ldots \ldots 3$

Jagannadham Venkata Pakala and Thomas Stephen Shores, On

compactly packed rings $\ldots \ldots \ldots \ldots \ldots \ldots \ldots \ldots \ldots$

Andrew Pletch, Strong completeness in profinite groups

Wilbur Carrington Whitten, Inverting double knots

James Juei-Chin Yeh, Existence of strong solutions for stochastic

differential equations in the plane 